\title{
Export policies for multi-domain WDM networks
}

\author{
Manolova, Anna Vasileva; Ruepp, Sarah Renée
}

Published in:

Proceedings OFC/NFOEC

Publication date:

2010

Document Version

Publisher's PDF, also known as Version of record

Link back to DTU Orbit

Citation (APA):

Manolova, A. V., \& Ruepp, S. R. (2010). Export policies for multi-domain WDM networks. In Proceedings OFC/NFOEC (pp. NWA5)

\section{General rights}

Copyright and moral rights for the publications made accessible in the public portal are retained by the authors and/or other copyright owners and it is a condition of accessing publications that users recognise and abide by the legal requirements associated with these rights.

- Users may download and print one copy of any publication from the public portal for the purpose of private study or research.

- You may not further distribute the material or use it for any profit-making activity or commercial gain

- You may freely distribute the URL identifying the publication in the public portal

If you believe that this document breaches copyright please contact us providing details, and we will remove access to the work immediately and investigate your claim. 


\title{
Export Policies for Multi-Domain WDM Networks*
}

\author{
Anna Manolova, Sarah Ruepp \\ DTU Fotonik, Department of Photonics Engineering, Danish Technical University, 2800 Kgs. Lyngby Danmark \\ \{anva,srru\}@fotonik.dtu.dk
}

\begin{abstract}
We analyze the performance of six export policies for a multi-domain routing protocol in WDM networks. We show that providing many AS-disjoint paths for survivability and load-balancing does not necessarily guarantee the lowest connection blocking. OCIS codes: $060.4251,060.4257$
\end{abstract}

\section{Introduction}

Applying Traffic Engineering (TE) methods in the process of connection provisioning is a must nowadays, considering the never ceasing demand for dynamic service provisioning with strict and diverse Quality of Service (QoS) requirements. The IETF's framework for Generalized Multi-Protocol Label Switching (GMPLS) [1] has established itself as a preferable technology for dynamic connection provisioning and TE within a single domain. With the development of more complex services stretching across domain boundaries the need for multi-domain TE and QoS provisioning has emerged as a hot research topic in both academia and industry. Several solutions for multi-domain routing [2-4] have been proposed and evaluated against the requirements for multi-domain TE and QoS provisioning in GMPLS networks [5]. Recently, a novel modification of the Border Gateway Protocol (BGP) [6] for multi-domain TE in WDM networks has been proposed in [7, 8]. The proposal is based on several extensions of the protocol, whose aim is to integrate the protocol within the GMPLS framework and to eliminate its most significant problems, when it is used for multi-domain routing in multidomain WDM transport networks [7]. One of the strongest characteristics of the proposal in [7] (referred to as Enhanced BGP) is the policy enforcement, which is inherent from the standard BGP.

A main feature of the GMPLS-controlled networks is the ability to provide resilience against failures in a distributed manner. For WDM transport networks this is of paramount importance due to the huge amount of affected traffic during failure events. In a multi-domain network the establishment of protection paths (which need to be link disjoint to the working paths [9]) is hindered by the strong privacy preservation policies between domains, which do not allow explicit topology information to leak out of the domain. A possible approach is to provide AS-disjoint paths which require the complex link-disjoint computations to be performed only in the source and the destination domains.

In this paper we propose several export polices for the Enhanced BGP protocol and evaluate their effect on the protocol overhead, the network performance and the ability of the protocol to provide AS-disjoint paths to destinations for load balancing and survivability support.

\section{Enhanced BGP for Multi-domain Routing in GMPLS Networks}

BGP has been the protocol of choice for multi-domain routing in the Internet for nearly 30 years and is a very well established tool for policy control between network domains. After analyzing the main drawbacks the standard BGP experiences when applied in connection oriented networks such as GMPLS-controlled networks, the authors of [7] propose an enhancement to the protocol. The operation of the Enhanced BGP can be seen on Fig. 1. The 3 main extensions are: i) distribution of an aggregated end-to-end TE metric per path per destination, ii) dissemination of multiple paths by disabling the path selection procedure of BGP and leaving only the path dissemination function active, iii) distribution of a Border-node sequence per path per destination, giving enhanced control to the head-end of a connection on the path. These features make the enhanced protocol very suitable for GMPLS-controlled WDM transport networks. The end-to-end TE metric is used as the only path selection criteria at the time of connection request and the border-node sequence is useful for providing explicit ERO for the RSVP-TE PATH messages during connection establishment and for eliminating the pathdependency problem, typical for the standard BGP operation. The specific operation of the protocol provides path diversity by distributing many paths per destination, which is beneficial for load balancing and survivability support. With the Enhanced BGP protocol, nodes can choose different paths peer destination at different times by practically achieving load balancing among the inter-domain links in the network, whose utilization is often unbalanced [7].

\footnotetext{
* The work described in this paper was carried out with the support of the BONE-project ("Building the Future Optical Network in Europe"), a Network of Excellence funded by the European Commission through the 7th ICT-Framework Programme.
} 


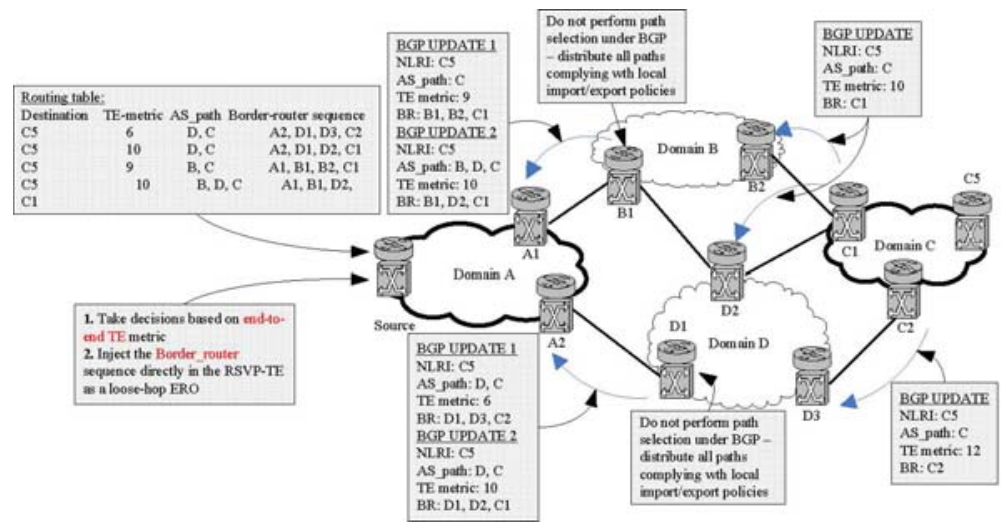

Fig. 1 Enhanced BGP operation

\section{Export policies}

A very important aspect of the Enhanced BGP protocol is the applied export policies which regulate the amount of distributed paths in the network. Furthermore, they greatly affect the scalability of the protocol. In a big network, with many inter-domain links and many BGP speakers, distributing every possible path will create enormous overhead. Thus, the amount of exported paths needs to be carefully controlled. Different export policies can be applied. Depending on the local TE goals (e.g. avoid paths which use many local resources) and the negotiated inter-domain agreements (e.g. do not export more than 3 paths for a given reachable destination to a neighbour) different set of rules can be applied in each BGP speaker. We propose and evaluate the performance of 6 different export policies:

- Policy 1: Export a path with longer AS_path attribute only if the path is AS-disjoint to all of the already distributed paths.

- Policy 2: Export only the first 5 of the already received paths.

- Policy 3 - exclusive: Export only paths which are AS-disjoint to all paths distributed so far.

- Policy 3 - inclusive: Export paths which are AS-disjoint to at least one path distributed so far.

- Policy 4 - exclusive: Export only paths which are Border-router-disjoint to all paths distributed so far.

- Policy 4 - inclusive: Export paths which are Border-router-disjoint to at least one path distributed so far.

The first policy aims at providing disjoint paths to destinations and at the same time minimize the path length (i.e. the end-to-end delay). The second policy aims only at minimizing the amount of distributed paths for improved protocol scalability. The goal of evaluating the last four policies is to see if loosening up the tight restrictions in the number of distributed paths will improve the network performance. The exclusive policies have the tendency to distribute less paths since a path needs to be disjoint to all previously distributed paths. When applying the inclusive policies, more paths are distributed which increases the chances for finding ASdisjoint pairs of paths. For scalability reasons only the first 20 paths are distributes under the inclusive policies.

\section{Simulation results}

The efficiency of the proposed export policies has been evaluated via simulations, performed on the event driven simulator OPNET [10]. The tested multi-domain topology is the COST 266 Pan-European topology [11], where each country represents one domain. The network has 22 domains connected via 42 bidirectional interdomain links. Each domain has between 1 and 4 randomly placed source/destination nodes.

Fig. 2 illustrates the ability of the policies to provide AS-disjoint paths to destinations, which is needed for improved network performance under failure scenarios. The results are presented in 5 categories: 1) source/destination pairs which do not have AS-disjoint paths between them, 2) which have only one AS-disjoint pair, 3) two AS-disjoint pairs, 4) three AS-disjoint pairs and 5) four or more AS-disjoint pairs of paths. Note that the amount of AS-disjoint paths in the border-nodes is not considered in the results, since source routing is used for connection establishment in GMPLS networks. As expected, the exclusive policies provide the least amount of AS-disjoint paths, whereas the inclusive policies provide the biggest amount of AS-disjoint paths. Fig. 3 illustrates the amount of BGP Update messages needed for distributing the paths and the average amount of paths per destination. The typical trade-off between protocol overhead and distributed information is obvious the more paths are disseminated, the better the chance for having more AS-disjoint pairs of paths, the more overhead is generated in the network. Considering the presented results, the inclusive policies provide the best basis for improved network performance under failure situation since more AS-disjoint paths can be identified and used. This comes at the expense of more than double the overhead, compared to Policy 1 and Policy 2.

Fig. 4 presents the connection blocking ratio versus the normalized input load per node for failure-free network operation where source nodes use all available paths per destination for connection establishment. Paths 
are chosen at the time of request based on the end-to-end TE metric, which is updated regularly. Despite the ability of the inclusive policies to provide more paths per destination their performance is worst. This is due to the fact that the provided paths are longer (see Fig. 5) which increases the probability for resource contention. Even though Policy 1 and Policy 2 do not necessarily provide AS-disjoint paths for all source/destination pairs they do achieve the best balance between protocol overhead and connection blocking ratio.



Fig. 2 Percentage of source/destination pairs with AS-disjoint paths for different categories and export policies

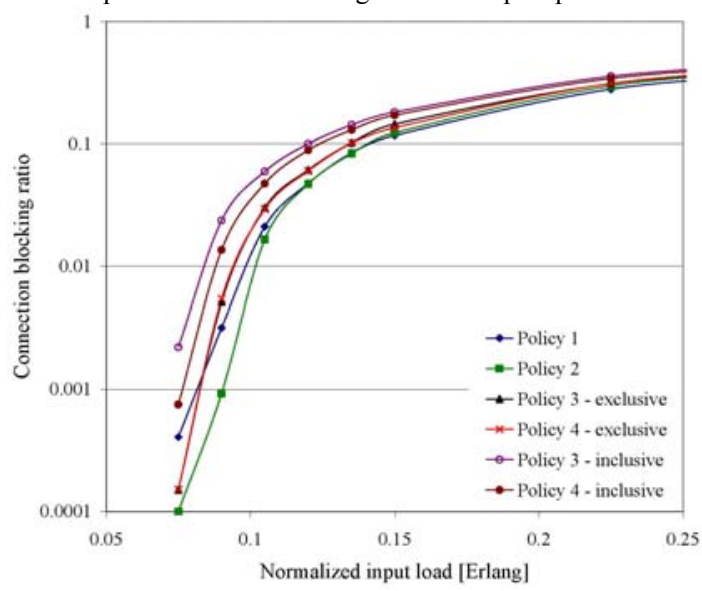

Fig. 4 Connection blocking ratio versus normalized input load per node for all tested polices

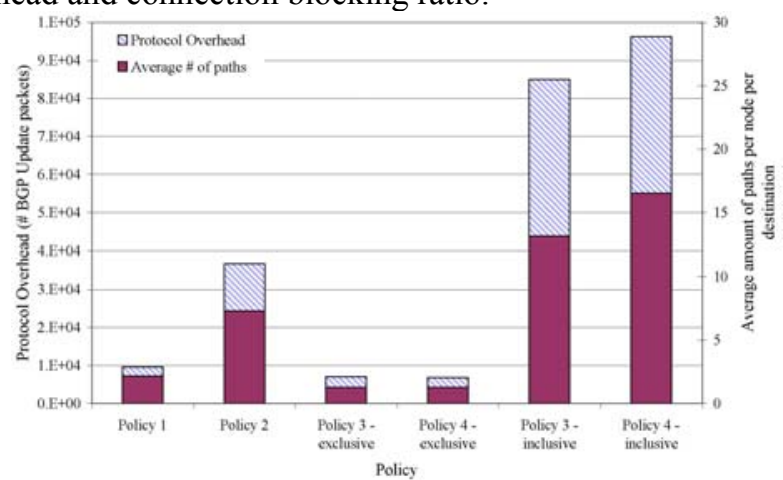

Fig. 3 Protocol overhead and average amount of paths per destination per node for all tested policies

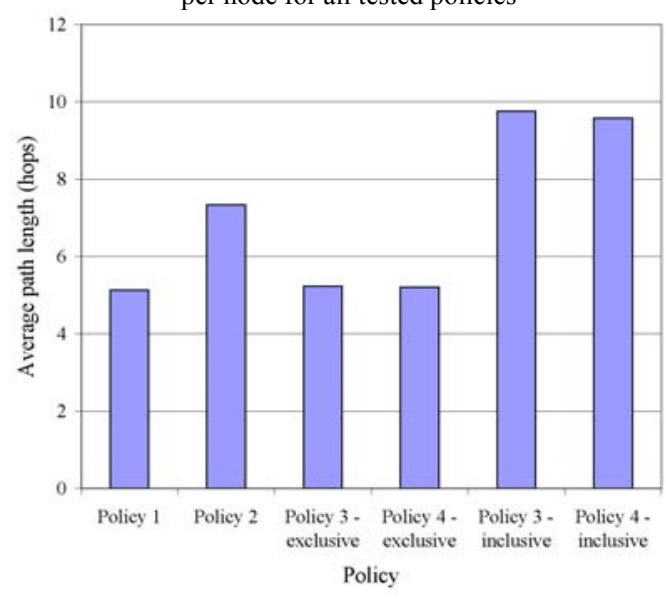

Fig. 5 Average path length per policy

\section{Conclusion}

In this paper we propose and evaluate the performance of six export policies for a recently suggested BGP enhancement for GMPLS multi-domain networks. Our results show that providing many paths per destination does not necessarily guarantee lowest connection blocking probability. On the other hand, the ability to provide AS-disjoint pairs of paths is improved, which is beneficial for survivability support. When choosing an export policy the trade-off between routing protocol overhead, supported survivability and average path length per destination, as well as the general multi-domain topology and the connectivity must be carefully considered. For survivability support, we recommend applying policies, which distribute many paths, i.e. the inclusive types, whereas for improved scalability we recommend policies which restrict the amount of distributed paths.

\section{References}

[1] E.Mannie, "Generalized Multi-Protocol Label Switching (GMPLS) Architecture", RFC 3945.

[2] A.Farrel, "A Path Computation Element (PCE)-Based Architecture", RFC 4655.

[3] The OIF Forum, "External Network-Network Interface (E-NNI) OSPF-based Routing", Jan. 2007.

[4] M.Blanchet, F.Parent, B.St-arnaud, “Optical BGP (OBGP): Inter AS Lightpath Provisioning,” IETF, ietf-draft-parent-obgp-01.txt, 2001.

[5] A. Farrel, J.-P. Vasseur, “A framework for Inter-domain Multi-protocol Label Switching Traffic Engineering”, RFC 4726, 2006

[6]Y. Rekhter, T. Li, S. Hares, “A Border Gateway Protocol 4 (BGP-4),” RFC 4271, 2006.

[7] A. Manolova, S. Ruepp, J. Buron, L. Dittmann, "On the efficiency of BGP-TE Extensions for GMPLS Multi-domain Routing", In Proc. of the $13^{\text {th }}$ Conference on Optical Network Design and Modelling : ONDM 2009 IEEE.

[8] A. Manolova, S. Ruepp, L. Dittmann, "Performance comparison of Multi-domain Routing Schemes in GMPLS networks with BGP”, In Proc. of 17th International Conference on Photonics in Switching 2009, IEEE Xplore.

[9] J.-P. Vasseur, M. Pickavet, P. Demeester, "Network Recovery - Protection and Restoration of Optical, Sonet-SDH, IP and MPLS", Morgan Kaufmann publishers, ISBN: 0-12-715051-x.

[10] OPNET Modeler, http://www.opnet.com

[11] R. Inkret et al. "Advanced Infrastructure for Photonik Networks," Extended final report of COST Action 266, available at http://www.ufe.cz/dpt240/cost266/. 